\title{
The Improved NSGA - II Based on Reverse Learning Mechanism Qiong Yuan ${ }^{1,2, a^{\star}}$, Guangming Dai ${ }^{1, b}$ \\ ${ }^{1}$ School of Computer Science ,China University of Geosciences, Wuhan,430074,China \\ ${ }^{2}$ Computer and Science Institute,Wuhan Donghu University, Wuhan,430212, China \\ aemail:yqszb@126.coml, bemail:gmdai@cug.edu.cn
}

Keywords: Multi-objective optimization; The NSGA-II algorithm;Reverse learning mechanism

\begin{abstract}
In this paper,According to the shortages of the NSGA - II algorithm in terms of the simulated binary crossover (SBX) operator, the speed of convergence and the diversity performance,The reverse learning mechanism (RLM) is applied to the initialization and evolutionary process of the NSGA-II,And introducing an improved arithmetic crossover operator.Through the series of ZDT test functions in two aspects of convergence and diversity evaluation, it show that the improved NSGA - II algorithm on the convergence speed, convergence and diversity is better than the NSGA - II algorithm.
\end{abstract}

\section{Introduction}

In 2002, Deb et al., in view of the disadvantages of NSGA, and put forward the improved algorithm NSGA-II. NSGA-II is a genetic algorithm based on Pareto optimal concept. Compared with NSGA, NSGA-II made the following improvements[1]:

(1)Using the method of rapid non dominated sorting, it reduced the computational complexity from $O\left(k N^{3}\right)$ down to $O\left(k N^{2}\right)$, where $\mathrm{k}$ is the number of target, and $\mathrm{N}$ is the number of individuals in the population.

(2) Adopting the crowding distance to maintain the diversity of population.

(3) The introduction of the elite strategy to prevent the loss of better solution.

Experimental results proved that the NSGA-II is superior to the other several representative algorithms. But the SBX (Simulated Binary Crossover) crossover operator has relatively weaker performance. To some extent, it limit the search performance of the algorithm. In addition, the NSGA-II in terms of the speed of convergence and the diversity of population remains to be improved.

\section{Reverse Learning Mechanism}

Reverse learning model was first proposed by Hamid R.Tizhoosh [2][3][4]. It has been proved to be an effective heuristic learning method, and successfully applied to the differential evolution algorithm[2][3][4], particle swarm optimization algorithm[5][6]to solve the unconstrained optimization problems, and some noise optimization problems.

The basic idea of reverse learning is: For a given problem, we should assess the current solution $X$, also want to evaluate the current solution of the reverse solution $X^{*}$. In the hope to find a better solution that reduces the distance between it and the optimal solution.

When $X$ is a multi-dimensional vector, it can calculate the inverse solution in the following way: Assume that $X=\left(x_{1}, x_{2}, \ldots, x_{D}\right)$ is a solution vector in a $D$ dimension space, where $x_{i} \in\left[L_{i}, U_{i}\right], \forall i \in\{1,2, \ldots, D\}, U_{i}$ and $L_{i}$ respectively represent lower and upper bounds of the ith dimension variable in the solution space. Then the reverse solution $X^{*}=\left(x_{1}^{*}, x_{2}^{*}, \ldots, x_{D}^{*}\right)$ of $X$ is defined as : $x_{i}^{*}=L_{i}+U_{i}-x_{i}$. 
Lei Peng et al. applied reverse learning mechanism in population initialization process and got the better result[7]. Because it considered not only the population $P$, but also the reverse population $P^{*}$. So, compared to single random initialization, there are more probability to close to the optimal target problem. Reverse learning initialization process is as follows:

(1) Randomly initializing the individuals of population $P$ :

$$
\begin{aligned}
& x_{i j}(0)=\operatorname{rnd}(0,1)\left(U_{j}-L_{j}\right)+L_{j} \\
& i=1,2, \ldots, N ; j=1,2, \ldots, D
\end{aligned}
$$

Where $N$ is the size of population, $D$ is the dimensionality of the solution space, $x_{i j}(0)$ is the jth dimensional variable value of the ith individual in the initial population P. $U_{j}$ and $L_{j}$ respectively represent upper and lower bounds of the ith dimension variable in the solution space.

(2) Calculating the individuals in the reverse population $P^{*} . x_{i j}^{*}(0)$ is the reverse variable value of $x_{i j}(0)$, that is the jth dimensional variable value of the ith individual in population $P^{*} \cdot x_{i j}^{*}(0)$ is calculated as follows: $x_{i j}^{*}(0)=L_{j}+U_{j}-x_{i j}(0)$.

(3) Choosing $\mathrm{N}$ best individuals as the initial population from population $P$ and reverse population $P^{*}$.

\section{Reverse Learning-based Evolution}

In this paper, reverse learning mechanism was applied to the evolutionary process.In the process of each generation evolution, to population $P$,calculating the reverse population $P^{*}$, and choosing $\mathrm{N}$ best individuals from population $P$ and reverse population $P^{*}$ as the next generation evolutionary population. However, taking into account the later stage of the algorithm, population $P$ has formed certain regularity, closing to the optimal solution area. Therefore, in the later stage of the algorithm, solving reverse population means no sense and slows down the speed of the algorithm.So, this paper designed the following method: In the process of each generation evolution, to population $P$, using a certain probability $r^{*}$ to calculate the reverse population $P^{*}$. And in the process of evolution, $r^{*}$ is linear decreasing. That is:

$$
r^{*}=\text { max_ } r^{*}-\frac{g}{G}\left(\max \_r^{*}-\min _{-} r^{*}\right)
$$

Where $G$ is the maximum generation number,and $g$ is the current generation number. max_ $r^{*}$ and min_ $r^{*}$ respectively are the maximum and the minimum of $r^{*}$. So, the application of reverse learning mechanism can accelerate the convergence of the algorithm. At the same time, in order to maintain the diversity of population, in the process of evolution, when the population individual $x_{i j}(g)$ and the reverse population individual $x_{i j}^{*}(g)$ are mutual non domination, using a certain probability $r$ to accept $x_{i j}^{*}(g)$. In the same way, probability $r$ is also linear decreasing, that is:

$$
r=\text { max } \_r-\frac{g}{G}\left(\max \_r-\min \_r\right)
$$

Where max $\_r$ and min_r respectively are the maximum and the minimum of $r$.

The crossover operator is one of the most important genetic operation. In the process of crossover, the excellent individual's genes pattern is rapidly reproduced and spreaded in a population. It could help other individuals of the population to go forward in the direction of the optimal solution.Compared to the simulated binary crossover operator, the arithmetic crossover operator has better global searching ability, and can maintain the diversity of population better.

The arithmetic crossover operation is as follows: Set $X_{A}^{t}$ and $X_{B}^{t}$ respectively as the real number encoding of decision variables for the two individuals to crossover in the th generation. After the crossover, the corresponding decision variable values of two individuals are as follows: 


$$
\begin{aligned}
& X_{A}^{t+1}=\alpha X_{A}^{t}+(1-\alpha) X_{B}^{t} \\
& X_{B}^{t+1}=\alpha X_{B}^{t}+(1-\alpha) X_{A}^{t}
\end{aligned}
$$

Where $\alpha$ is the parameter.When $\alpha$ is a constant, it is called uniform arithmetic crossover at this time. Otherwise, it is called non uniform arithmetic crossover[8].

Considering that in the process of evolution, it is hoped that, the individual of the population which has better rank (rank has low value )and better distribution (dist has larger value), occupy a larger proportion of gene in the offspring. So, the paper design the following crossover operator coefficient:

$$
\alpha=\left\{\begin{array}{lc}
\frac{\text { B.rank }}{\text { A.rank }+ \text { B.rank }} & \text { A.rank } \neq \text { B.rank } \\
\frac{\text { A.dist }}{\text { A.dist }+ \text { B.dist }} & \text { A.rank }=\text { B.rank }
\end{array}\right.
$$

Where, A.rank represents non dominated sorting rank of the current generation of the individual A. A.dist represents crowding distance of the current generation of the individual $A$. So, in the prophase of the algorithm, better individual genes get better retention. So the algorithm has faster convergence speed. Meanwhile, In the later stages of the algorithm, better distribution individual genes get better retention. So improving the diversity of the algorithm.

Based on the above analysis, the improved NSGA-II algorithm are as follows:

/*Opposition-based population initialization*/

Random initialization of population $P$;

Calculate opposite population $P^{*}$;

Selecting $N$ fittest individuals from $P$ and $P^{*}$ as initial population;

$/ *$ End of Opposition-based population initialization*/

Evaluate initial population;

While(the halting criterion is not satisfied)

\{

Tournamenet Selections routines;

Arithmetic Crossover routines;

Polynomial Mutation routines;

Evaluate population $\mathrm{P}$;

If(rnd $\left.(0,1)<r^{*}\right)$

\{

Compute opposite population $P^{*}$;

Select PopSize fittest Individuals from $P$ and $P^{*}$;

\}

\}

\section{Numerical experiments}

In order to verify the improved NSGA-II algorithm performance, this article selects the classic series of ZDT (ZDT1,ZDT2,ZDT3,ZDT6) function as test functions.

Table 1 The dimensions in decision space and in object space on the test function

\begin{tabular}{|c|c|c|}
\hline Test function & The dimensions in decision space & The dimensions in object space \\
\hline ZDT1, ZDT2, ZDT3 & 30 & 2 \\
\hline ZDT6 & 10 & 2 \\
\hline
\end{tabular}

The evaluation standard of the test results uses the convergence and the diversity of the results.

The experiment parameters are set as follows:

The population size 100, Maximum generation number 200, Crossover probability 0.9, Mutation probability 0.1 .

The two upper and lower bounds of the probability on the improved NSGA- II: 
max $\_r^{*}=0.8$, min $\_r^{*}=0.5 ;$ max $\_r=1.0$, min $\_r=0.0$;

The experimental results are the mean of 10 times.

Table 2 convergence and diversity of the two algorithms on ZDT1

\begin{tabular}{|l|l|l|}
\hline \multirow{2}{*}{ Algorithm } & \multicolumn{2}{|c|}{ ZDT1 } \\
\cline { 2 - 3 } & \multicolumn{1}{|c|}{ Convergence } & Diversity \\
\hline NSGA-II & $0.00277211+0.00140097$ & $0.372788+-0.108156$ \\
\hline My NSGA-II & $\mathbf{0 . 0 0 1 1 1 1 8 1 + - 0 . 0 0 0 1 7 2 2 0 8}$ & $\mathbf{0 . 3 7 0 2 9 8 + - 0 . 0 8 1 5 0 2 9}$ \\
\hline
\end{tabular}

Table 3 convergence and diversity of the two algorithms on ZDT2

\begin{tabular}{|l|l|c|}
\hline \multirow{2}{*}{ Algorithm } & \multicolumn{2}{|c|}{ ZDT2 } \\
\cline { 2 - 3 } & \multicolumn{1}{|c|}{ Convergence } & Diversity \\
\hline NSGA-II & $0.00297525+-0.00223656$ & $0.386197+-0.0671831$ \\
\hline My NSGA-II & $0.000852262+-0.000107841$ & $0.417186+-0.10672$ \\
\hline
\end{tabular}

Table 4 convergence and diversity of the two algorithms on ZDT3

\begin{tabular}{|c|c|c|}
\hline \multirow{2}{*}{ Algorithm } & \multicolumn{2}{|c|}{ ZDT3 } \\
\hline & Convergence & Diversity \\
\hline NSGA-II & $0.00184749+-0.000186385$ & $0.685294+-0.0449552$ \\
\hline My NSGA-II & $0.00107189+-3.01321 \mathrm{e}-05$ & $0.58475+-0.0886773$ \\
\hline
\end{tabular}

Table 5 convergence and diversity of the two algorithms on ZDT6

\begin{tabular}{|l|l|c|}
\hline \multirow{2}{*}{ Algorithm } & \multicolumn{2}{|c|}{ ZDT6 } \\
\cline { 2 - 3 } & \multicolumn{1}{|c|}{ Convergence } & Diversity \\
\hline NSGA-II & $0.0192366+-0.00406833$ & $0.374652+-0.0639926$ \\
\hline My NSGA-II & $0.00486568+-0.00232125$ & $0.317342+-0.0793183$ \\
\hline
\end{tabular}

In order to facilitate a better comparison, Figure 1 to figure 4 are comparison charts of NSGA-II and improved NSGA-II program running after 150 generations of the results with the standard front.

From table 2 to table 5,the improved NSGA - II algorithm on the convergence performance is excellent.It's convergence on four test function is better than the NSGA-II algorithm.Meanwhile,The improved NSGA - II algorithm also has large improvement in terms of diversity.In addition to the diversity in the ZDT2 function lower than the NSGA - II, Other test functions are superior to the diversity of the NSGA - II.From chart 1 to chart 4,the convergence rate of the improved NSGA - II is better.So,it proved the validity of the improved algorithm.

\section{Conclusion}

Aiming at the deficiency of the NSGA-II algorithm,in this paper,it introduced reverse learning mechanism to the initialization and evolutionary process of the algorithm,And introduced the improved arithmetic crossover operator instead of the original simulated binary crossover operator.Finally,Getting an improved the NSGA-II algorithm.The experimental results show that:The improved NSGA-II algorithm has a different degree of improvement on the convergence speed, convergence and the diversity.

\section{Acknowledgement}

In this paper, the research was sponsored by the National Natural Science Foundation(Project No. 61472375) and Youth Fund Project of Wuhan Donghu University (Project No. 2014) . 


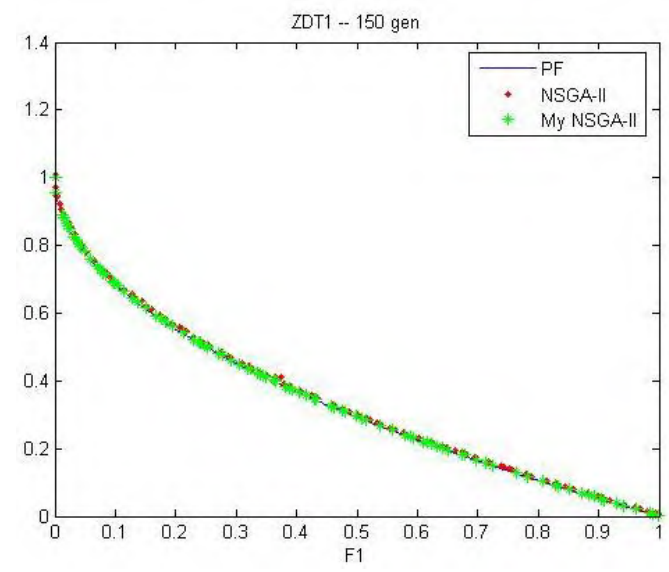

Chart 1 two algorithms on ZDT1with frontier

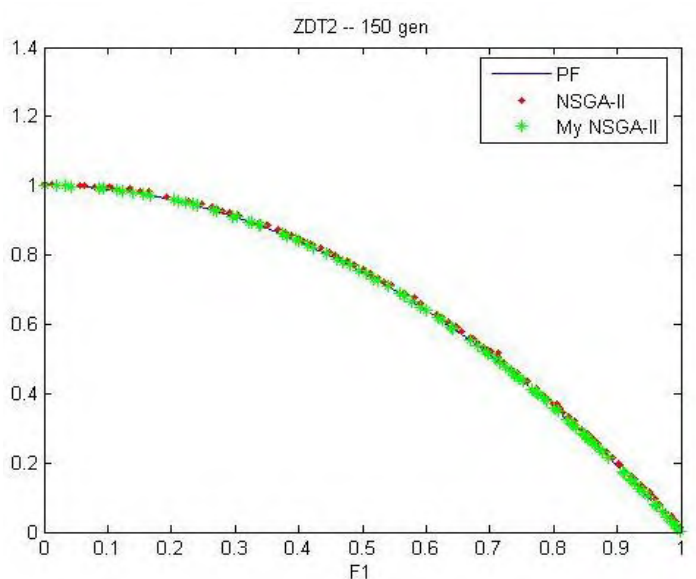

Chart 2 two algorithms on ZDT2 with frontier

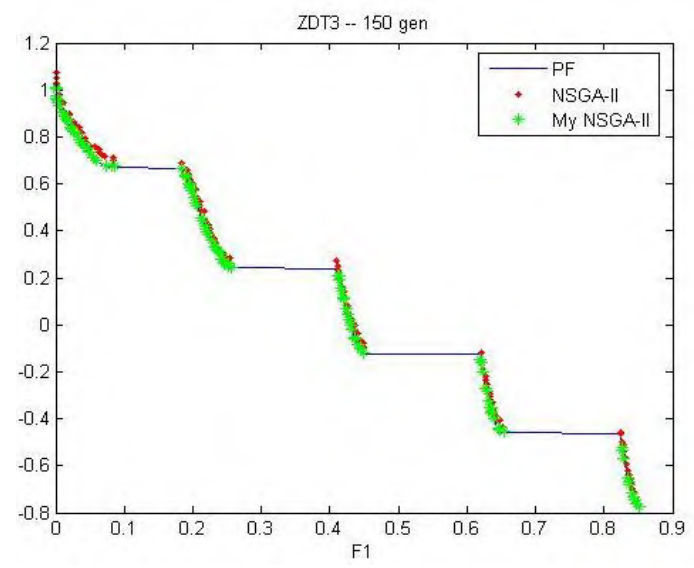

Chart 3 two algorithms on ZDT3 with frontier

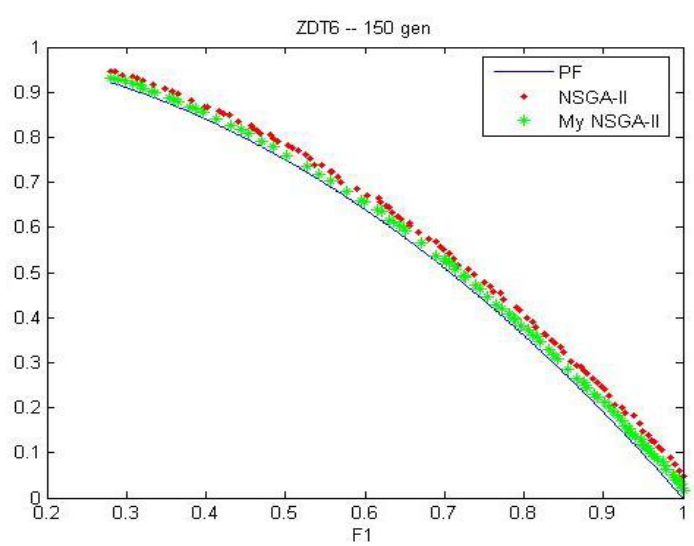

Chart 4 two algorithms on ZDT4 with frontier

\section{References}

[1] Q.Zheng. Elitist Nondominated Sorting Genetic Algorithm and Its Application[D].hangzhou : School of information science and engineering, zhejiang university, 2006.

[2]S.Rahnamayan,H.R.Tizhoosh,M.M.A.Salama.Opposition-based Differential Evolutional Algorithms[C]. In Proeeeding of the IEEE Congress on Evolutionary Computation,2006, 2010-2017.

[3]S.Rahnamayan,H.R.Tizhoosh,M.M.A.Salama. Opposition-based Differential Evolution for Optimization of Noisy Problems[C]. In Proeeeding of the IEEE Congresson Evolutionary Computation, 2007, 1865-1872.

[4]S. Rahnamayan, H. R.Tizhoosh,M. M. A. Salama. Opposition-Based Differential Evolution[J], IEEE Trans.Evolutionary Computation, VOL. 12, NO. 1, 2008, 64-79.

[5] H.Wang, Y. Liu, S. Zeng, H. Li,C. Li. Opposition-based Particle Swarm Algorithm with Cauchy Mutation[C]. In Proeeeding of the IEEE Congresson Evolutionary Computation, 2007, 4750-4756.

[6]H.Wang. Research on Hybrid Particle Swarm Algorithms with Cauchy Mutation[D].Wuhan: Computer college, China university of geosciences, 2008.

[7]Lei Peng, Yuanzhen Wang, Guangming Dai. A Novel Opposition-Based Multi-objective Differential Evolution Algorithm for Multi-objective Optimization[C]. ADVANCES IN COMPUTATION AND INTELLIGENCE Lecture Notes in Computer Science, 2008, Volume 5370/2008, 162-170.

[8]X.H.Liu,Y.S.Liu,G.Y.Zhang etc..Improvement of Multi-objective Optimization Algorithm NSGA-II[J].Computer engineering and application : 2005,15,73. 